\title{
Modular interfaces for on-line applications
}

\author{
RONALD R. MOURANT and JEFFREY W. SMITH \\ Department of Industrial Engineering \& Operations Research \\ Wayne State University, Detroit, Michigan 48202
}

\begin{abstract}
Analog signals, generated by BASIC R/T plot commands, are used to control a modular interface system. The interface system converts the analog signals to either BCD or decimal formulated pulses which are used to drive up to 10 relays. The system is coupled to a LAB 8/E minicomputer that permits precise timing of intersignal intervals as well as the simultaneous recording of many response variables.
\end{abstract}

Several successful systems for controlling on-line experiments have already been built (Barensten \& Lockard, 1973; Getty, 1975: Moise, Olson, \& Huston, 1974). However, these systems require the use of an assembly language or a special software system. In contrast, the system described below can activate relays and monitor inputs by simply using plot and analog-to-digital command statements associated with any real-time language. Since the modular system was interfaced to a $\mathrm{LAB} 8 / \mathrm{E}$ minicomputer. clock commands available through BASIC $R / T$ were readily available for timing stimuli durations and measuring response latencies. The highlevel language also enables students to have immediate access to use of the modules.

The system was also designed to accommodate a variety of experimental paradigms. The experimenter can easily program when a given number of relays should be activated. as well as monitor several input channels. The activation of relays can also be made to depend on some characteristic of a subject's response. The system contains (1) modules for generating pulses to control switch-operated devices, (2) modules for use with incoming signals, and (3) a warning light module. Each modular unit may be debugged separately and then tested as part of the system, as suggested by McLean in 1973.

\section{MODULES FOR CONTROLLING SWITCH-OPERATED DEVICES}

Switch-operated devices commonly controlled in online experiments include lights, buzzers, tachistoscopes, and slide projectors (Buckley \& Gillman, 1973). In order to operate these devices, the modular system contains 10 relays which may be operated either simultaneously or in rapid succession. The experimenter has the choice of a normally open or normally closed relay output. Pulse inputs to the relays are generated by tapping $x, y$. and $z$ analog outputs from a VC8-E display control. The display control is normally used to plot points on a Tektronix Model 602 oscilloscope. The $x$ signal from the display control is transmitted to an analog-to-BCD (binary code decimal) converter. This converter is wired so that commands which plot a dot on the $\mathrm{x}$ axis will generate combinations of pulses on Output Channels $1,2,4$, and 8 . Table 1 presents the BCD channels that are activated by various plot commands.

Actual conversion of the $x$ analog signal takes place immediately and is available for use upon receipt of a strobe signal. The strobe input is generated by the intensifying circuit of the display control ( $z$ output signal) and a small time delay (about 1 microsec). Use of points only after they become fixed prevents accidental relay activation by the analog $\mathrm{x}$ signal.

The output from the analog-to-BCD module may be connected to the latch input of a relay. In this mode the relay remains activated until the latch input is pulsed again. Timing may be done with the LAB 8/E's real-time clock (DK8-ES). Four separate relays may be controlled in this manner since there are four output channels on the analog-to-BCD converter.

By use of a BCD-to-decimal decoder module, up to 10 relays may be controlled. The module has four input channels $(1,2,4$, and 8$)$ and 10 output channels ( 1 through 10). Combinations of pulses on the four input channels (generated by a PLOT command) result in a pulse on one of the output channels. The decimal outputs may be connected to a digital display so that the experimenter may compare actual output with program commands.

Table 1

PLOT Commands and BCD Outputs

\begin{tabular}{ccccc} 
& \multicolumn{4}{c}{ Output Channels } \\
\cline { 2 - 5 } PLOT & 1 & 2 & 4 & 8 \\
\hline 0,0 & 0 & 0 & 0 & 0 \\
$.1,0$ & 1 & 0 & 0 & 0 \\
$.2,0$ & 0 & 1 & 0 & 0 \\
.3 .0 & 1 & 1 & 0 & 0 \\
.4 .0 & 0 & 0 & 1 & 0 \\
.5 .0 & 1 & 0 & 1 & 0 \\
$.6,0$ & 0 & 1 & 1 & 0 \\
$.7,0$ & 1 & 1 & 1 & 0 \\
$.8,0$ & 0 & 0 & 0 & 1 \\
$.9,0$ & 1 & 0 & 0 & 1 \\
\hline
\end{tabular}



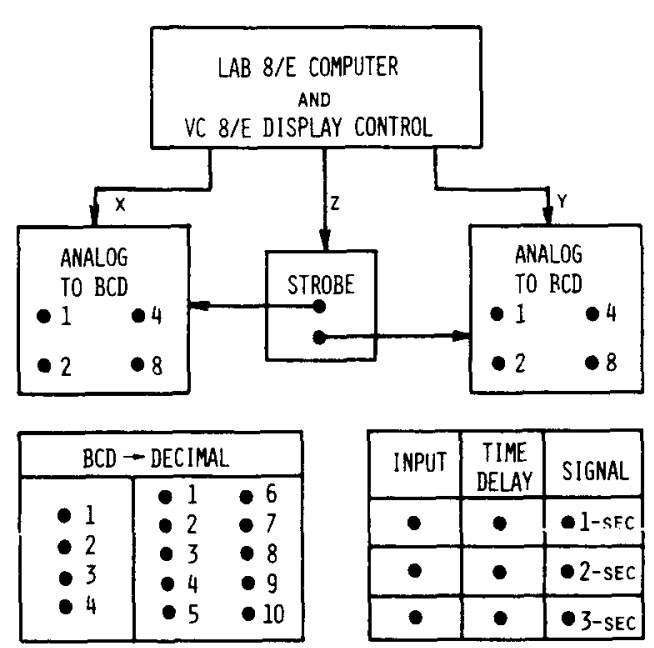

10-RELAYS

EITHER LATCH OR NORMAL INPUTS

Figure 1. System components for operating relays.

Before signals from either the analog-to-BCD converter or the $\mathrm{BCD}$-to-decimal decoder are sent to a relay, they may be inputted to a time delay module. The experimenter may choose between simply having the signal delayed for a specific time period $(1,2$, or $3 \mathrm{sec})$ or having an immediate output signal of approximately a $1-, 2-$, or 3-sec duration. The output from the delay module is connected to the regular input of a relay. The $1 \cdot, 2$-, or $3-\sec$ output signals are useful for advancing one, two, or three slides when using a Kodak Carousel projector. Use of the time delay module frees the computer from having to time the pulse duration; thus, the next program statement may be immediately executed.

Figure 1 is a diagram of the system components used for operating relays. Note that it is sufficient to sample the $\mathrm{x}$ analog signal to operate 10 relays. An additional 10 relays may be operated using the $y$ analog signal if another BCD-to-decimal decoder is added to the system.

\section{MODULES FOR INPUT SIGNALS}

Modules for use in connection with incoming signals include a time delay buffer, a decimal-to-BCD incoder, a voltage divider, reference voltages, and double ended limit detectors.

The time delay buffer requires that an incoming signal be present for at least $20 \mathrm{msec}$ before an output signal lasting $100 \mathrm{msec}$ is generated. This interface protects against false signals that may be generated by highfrequency noise. Miller and Polson (1972) describe a more sophisticated unit for incoming signals that provides for better noise protection.
If a large number of incoming channels are being used, then the decimal-to-BCD incoder module may be useful. This module codes 10 incoming channels into four, thus, the computer needs to sample only four channels.

The voltage divider module simply divides the incoming voltage by five. This enables incoming signals to be accepted by the analog-to-digital converter channels of the LAB 8/E. These channels require that a signal be restricted to $\pm 1 \mathrm{~V}$.

The reference voltage module provides 10 signals ranging from $+1.5 \mathrm{~V}$ to $-1.5 \mathrm{~V}$ in steps of $.25 \mathrm{~V}$. These signals may be connected to response buttons and then used as one input signal to the analog-to-digital converter. They provide an accurate method of determining what response button has been pressed.

The double ended limit detector is for use with \pm 5 . $\mathrm{V}$ dc signals. A window is generated by adjusting an upper and lower limit. Then, output signals may occur only when the input signal is in the "window." The limit detectors are useful for processing composite video and neurophysiological signals (Beatty \& Figueroa, 1975).

\section{Warning Light Module}

Because warning lights are often used in experiments, a separate module was built to control them. Any light may be plugged into the module. A normally on or normally off switch determines the state prior to the computer command. A variable intensity control is provided on the face of the module. The module also has three sets of relay contacts which are activated by the same signal that controls the warning light. Thus, it is possible to control other devices at the same time the warning light is turned on or off.

\section{Convenience Features}

A remote patch panel was constructed since it is common practice to locate switch-operated devices and subject response buttons some distance from the computer. The remote patch panel contains 50 channels that are duplicated at the other end of a $40-\mathrm{ft}$ cable. The channels may be used to control switches or to receive incoming signals. The panel results in a tidy laboratory and saves the experimenter time because it eliminates the use of long lengths of wire.

All inputs and outputs described use banana plug connectors (except the LAB 8/E analog-to-digital converters which have phone jack inputs). However, provisions have been made to accept signals from microphone, UHF, BNC, and phone plug connectors. Signals from these connectors are simply converted to banana plug outputs. The system has been successfully used in a number of experimental situations. 


\section{REFERENCES}

BARE NSTEN, R. I., \& LockARD, J. S. Minicomputer controller for a behavioral primate laboratory. Behavior Research Methods \& Instrumentation. 1973, 5, 111-113.

BeatTy. J., \& Figueroa, C. The detection and treatment of artifacts in computer-controlled neurophysiological experiments. Behavior Research Methods \& Instrumentation, 1975. 7. 179-182.

Buckley, P. B., \& Gillman, C. B. The slide projector as a computer-operated visual display. Behavior Research Methods \& Instrumentation, 1973, 5, 104-106.
GETTY, D. J. The PEPL system for control of experiments by a PDP.8 computer. Behavior Research Methods \& Instrumentation, 1975, 7, 131-136.

McLean, R. S. Hardware debugging for on-line psychologists. Behavior Research Methods \& Instrumentation, 1973, 5. 107-110.

Miller. R. F., \& Polson, P. G. An interface for transmitting discrete response information to a computer. Behavior Research Methods \& Instrumentation, 1972, 4, 331-333.

Moise, S. L.. JR., Olson, D. E., \& Huston, S. W. An automated system for primate instruction for behavioral neurophysiology. Behavior Research Methods \& Instrumentation, 1974, 6, 557-564. 